\title{
ON SYMMETRY-CONCERTED ORGANOMETALLIC ANALOGUES OF THE ETHYLENE DIMERIZATION
}

\author{
S. Roszak, H. Chojnacki \\ Institute of Physical and Theoretical Chemistry, Technical University of Wrocław \\ Wyb. Wyspiańskiego 27, 50-370 Wrocław, Poland \\ AND K. BALASUBRAMANIAN \\ Department of Chemistry and Biochemistry, Arizona State University \\ Tempe, Arizona 85287-1604, USA \\ (Received March 22, 1995) \\ Dedicated to Professors Krzysztof Pigoń, Józef W. Rohleder \\ and Zdzisław Ruziewicz on the occasion of their 70th birthday* \\ A theoretical study of the $C_{2 v}$ symmetry-concerted reaction is presented \\ for the addition of ethylene and molybdenum dimer. The potential curves \\ corresponding to different self-consistent field closed shell configurations are \\ studied. A comparison of ethylene dimerization and ethylene- $\mathrm{Mo}_{2}$ formation \\ reactions indicates close similarities and applicability of the Woodward-Hoff- \\ mann model, developed originally for olefins, to organometallics.
}

PACS numbers: 31.20 .Ej

\section{Introduction}

Interaction of transition metal dimers which possess multiple bonds with molecules such as ethylene and butadiene could provide a novel insight into organometallic analogues of the Diels-Alder reaction [1-2]. Such reactions can also offer novel models for testing symmetry-concerted organometallic reactions that conform to the Woodward-Hoffmann model of such reactions. Such studies provide

*Submitted on invitation of the Institute of Physical and Theoretical Chemistry, Technical University of Wrọctaw, Wrocław, Poland. 
insight into potential organometallic analogue of the symmetry-concerted ethylene dimerization as

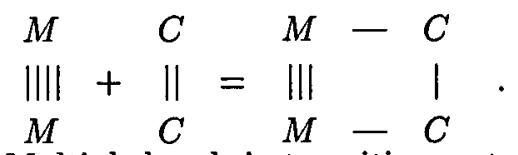

Multiple bonds in transition metal dimers composed of open shell transition metal atoms such as $\mathrm{Zr}_{2}, \mathrm{Mo}_{2}, \mathrm{~W}_{2}, \mathrm{Cr}_{2}$ offer a large number of low-lying electronic states that can interact with unsaturated organic compounds in a symmetry-concerted manner. Such reactions have been studied previously for ethylene with $\mathrm{Mo}_{2}$ [3], $\mathrm{Zr}_{2}$ [4] and $\mathrm{Cu}_{2}$ [5], applying rigorous quantum chemical methods. However, considerations regarding the symmetry-concerted reactions performed by Woodward and Hoffmann [6] lead to successful general rules, even if one applied a much lower level of the theory.

In this work we study the reaction of $\mathrm{MO}_{2}$ with ethylene (and the ethylene dimerization for comparison purposes) applying a simple model of self-consistent field (SCF) adiabatic potential curves based on interacting closed shell configurations. The presented approach is equivalent to the one developed by Woodward and Hoffmann [7] for reactions of cyclization of olefins. The above reaction has been studied previously at the advanced ab initio level (CASSCF/FOCI) with the inclusion of correlation energy [3], and those results serve as a test for the current qualitative picture. Furthermore, in the current investigation a comparison is made with the analogous 2 ethylene $\rightarrow$ cyclobutane ring closure reaction which is studied here at the same level of the theory.

\section{Method and computational details}

The presented calculations were performed using the closed-shell SCF method. Potential surfaces were calculated for different configurations assumed for the closed shell wave function. The optimized ground state geometries for the ethylene dimerization and $\mathrm{Mo}_{2}$-Eth complex formation reaction pathways at the SCF level have been utilized in the study. All studies performed here were made using relativistic effective core potentials (RECPs), which retained the outer $4 s 4 p 4 d 5 s$ shells of Mo [8] and $2 s 2 p$ shells for the carbon atom [9]. The basis set for Mo was prepared from the work of LaJohn et al. [8] by decontracting the most diffuse Gaussians resulting in a valence $(3 s 3 p 2 d)$ basis set. The basis set for carbon was taken from Pacios and Christiansen [9], but we leave the most diffuse functions uncontracted. The carbon basis set was supplemented with six-component $3 d$ Gaussian functions adopted from Dunning and Hay [10], leading to a $(2 s 2 p 1 d)$ basis set. For hydrogen, Van Duijneveldt's [11] (4s) basis set was employed. The calculations were performed using the GAMESS package of codes [12].

The geometry of the $\mathrm{Eth}_{2}$ complex was optimized assuming the $C_{2 v}$ symmetry along the reaction pathway (Fig. 1). The computed cyclobutane geometry (C-C $1.56 \AA, \mathrm{C}-\mathrm{H} 1.09 \AA<\mathrm{HCH} 107.7$ degree) agrees with experimental structure (C-C $1.56 \AA, \mathrm{C}-\mathrm{H} 1.09 \AA,<\mathrm{HCH} 114 \pm 8$ degree) [13]. The C-C bond distance for ethylene (in the dissociated structure) of $1.35 \AA$ agrees with the corresponding experimental value of $1.322 \AA[13]$. Values of $\mathrm{HCH}$ angles in ethylene and cyclobutane indicate the expected change from $s p^{2}$ to $s p^{3}$ orbital hybridization on 


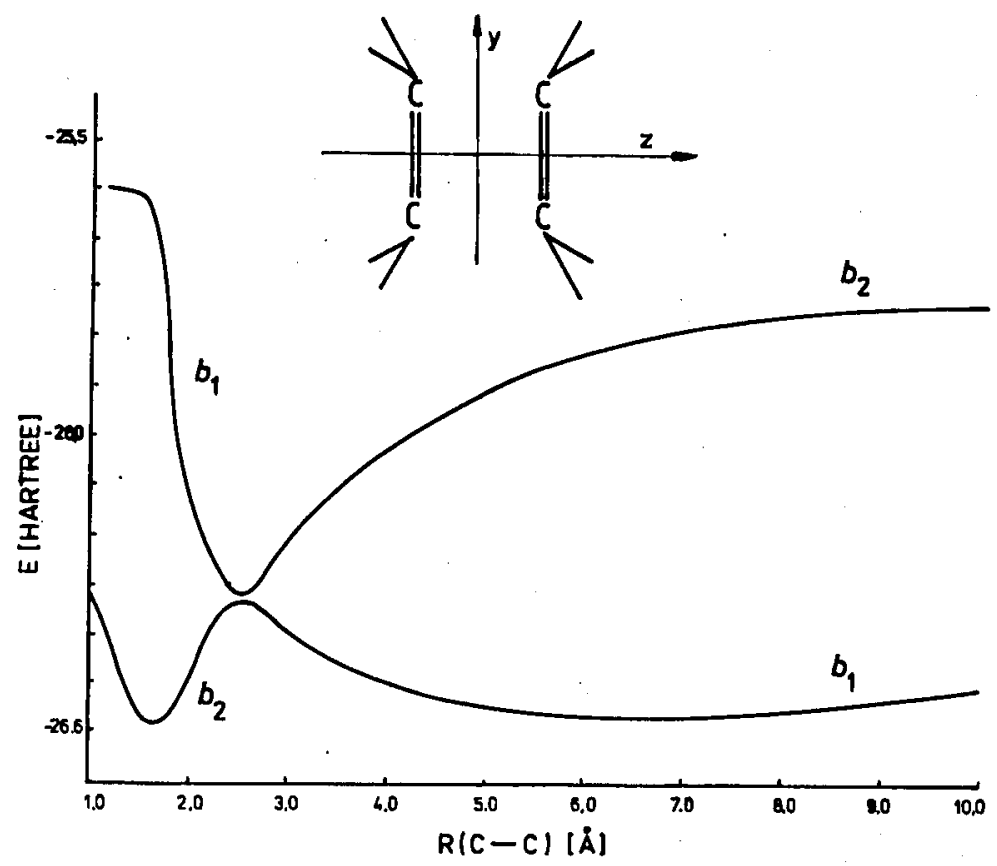

Fig. 1. SCF potential curves for the ethylene dimerization reaction.

carbon corresponding to conversion of the $\mathrm{C}=\mathrm{C}$ double bond into a single bond. The optimized geometry for the $\mathrm{Mo}_{2}-$ Eth reaction pathway has been adopted from Ref. [3].

The complete active space self-consistent field (CASSCF) calculations performed for the ethylene dimerization included the highest occupied and the lowest unoccupied molecular orbitals in the active space.

\section{Results and discussion}

The $C_{2 v}$ symmetry-concerted reaction (Fig. 1) of the ethylene dimerization leads to cyclobutane. The SCF ground state configuration $\left({ }^{1} A_{1}\right)$ of cyclobutane is $4 a_{1}^{2} 2 a_{2}^{2} 3 b_{1}^{2} 3 b_{2}^{2}$. However, the lowest energy for the dissociated cyclobutane to two ethylenes corresponds to a different $4 a_{1}^{2} 2 a_{2}^{2} 4 b_{1}^{2} 2 b_{2}^{2}$ configuration. The continuation of the cyclobutane equilibrium configuration ((core) $\left.b_{2}^{2}\right)$ along the dissociation pathway leads to the electronic excited state of the dissociated system. The excited state of cyclobutane described by the (core) $b_{1}^{2}$ configuration correlates with the ground state of two separated ethylenes. Such correlations, due to the "non-crossing rule", lead to a potential barrier along the reaction path (Fig. 1). The above behavior of potential curves in a multiconfiguration approach, is predicted more accurately since it allows one to treat both equilibrium and dissociated configurations. The calculation for the ethylene dimerization within the CASSCF formalism, including (core) $b_{1}^{2}$ and (core) $b_{2}^{2}$ configurations in the active space, leads to almost pure single-determinantal configurations for reactants 
and product, and gives $44 \%$ (core) $b_{2}^{2}+56 \%$ (core) $b_{1}^{2}$ wave function at the transition state. Simple HOMO/LUMO CASSCF calculations significantly lower the barrier to $23 \mathrm{kcal} / \mathrm{mol}$, although qualitative conclusions derived from the adiabatic SCF curves are correct.

The question arises about the shapes of the adiabatic SCF curves, when one of the ethylenes is replaced by the metal dimer having a multiple bond. The chosen example of $\mathrm{Mo}_{2}$ offers a variety of bonds (sixtuple Mo-Mo bond [14]) and a variety of possible configurations. Due to a number of low-lying electronic states of $\mathrm{Mo}_{2}$, the reaction arises from the interaction of the ground state of ethylene with different electronic states of $\mathrm{Mo}_{2}$. The ground electronic state of the complex has a (core) $b_{2}^{2} b_{1}^{2} a_{1}^{2}$ configuration, while for the dissociated structure, the lowest energy corresponds to (core) $b_{1}^{2} a_{2}^{2} a_{1}^{2}$. Due to the "non-crossing rule" the ground state potential surface exhibits a potential barrier similar to the dimerization of ethylene. The template of $\mathrm{Mo}_{2}$ states interacting with ethylene leads to complicated potential surfaces especially in the excited electronic states (Fig. 2). Some low-lying excited states of $\mathrm{Mo}_{2}$ offer patential barrierless reaction pathways. These observations agree qualitatively with more rigorous CASSCF and FOCI calculations [3].

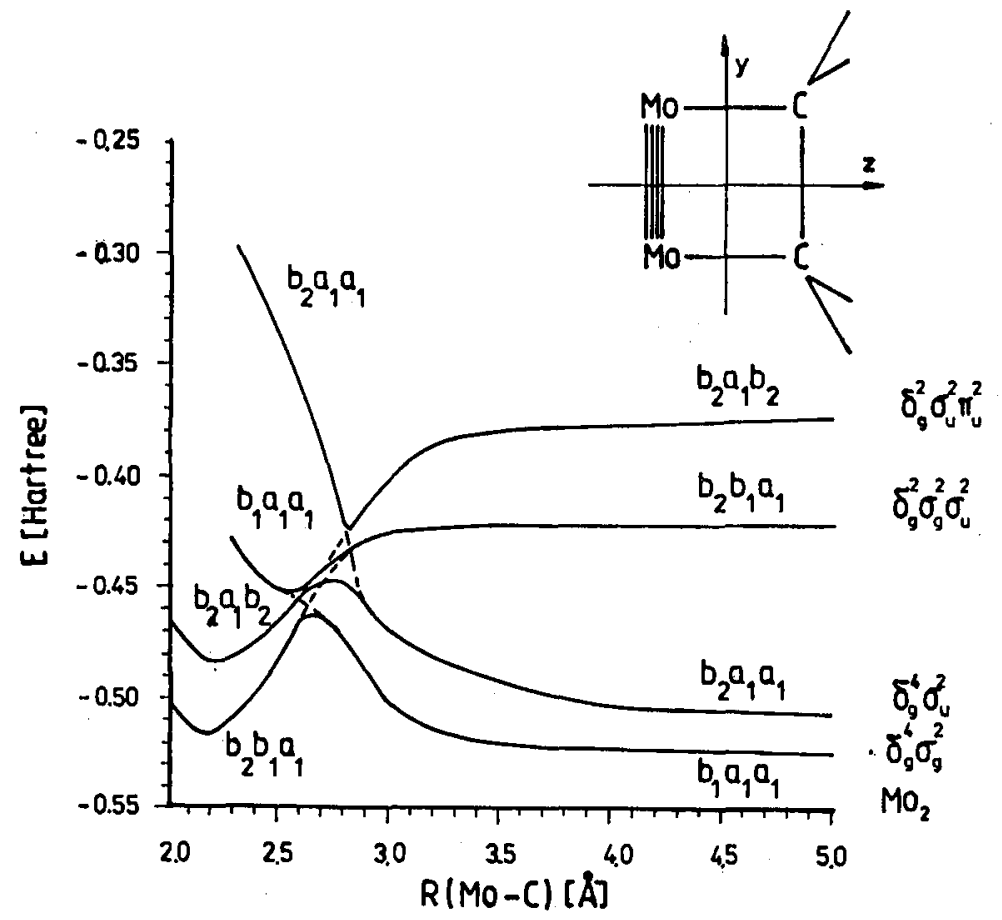

Fig. 2. Closed shell SCF potential curves for the reaction of ethylene with $\mathrm{Mo}_{2}$ (the calculated values of energy are $-147+E$ ). 


\section{Conclusions}

Differences in the composition of the molecular wave function for cyclobutane and the dissociated ethylenes lead to the potential barrier along the reaction pathway. Replacement of one ethylene molecule by the $\mathrm{Mo}_{2}$ dimer offers a system rich in low-lying electronic states. In the studied test case of $\mathrm{Mo}_{2}-\mathrm{Eth}$, the ground state wave function also changes its character along the reaction pathway (the Slater determinant differs by molecular orbitals carrying different symmetry) in full analogy to the ethylene dimerization. This also leads to an avoided crossing between the ground and excited potential surfaces and in consequence to a potential barrier. However, some low-lying excited states of the $\mathrm{Mo}_{2}$ dimer, lead to excited state reaction paths without barrier. Both conclusions agree with large scale CASSCF/FOCI calculations including correlation energy [3], indicating the usefulness of the presented approach.

\section{Acknowledgment}

This research was supported in part by the U.S. Department of Energy under Grant DE FG0286R13558 and by TU Wroclaw research project No. 341-336.

\section{References}

[1] E.L. Muetterties, T.N. Rodin, E. Band, C.F. Brucker, R.W. Pretzer, Chem. Rev. 79, 91 (1979).

[2] P. Heimbach, R. Traunmuller, Chemie der Metall-Olefin-Komplexe, Verlag Chemie, Weinheim 1970, and references cited therein.

[3] S. Roszak, K. Balasubramanian, Inorg. Chem. 33, 4169 (1994).

[4] S. Roszak, K. Balasubramanian, J. Phys. Chem., in press.

[5] S. Roszak, K. Balasubramanian, Chem. Phys. Lett. 231, 18 (1994).

[6] R.B. Woodward, R. Hoffmann, Angew. Chem. Int. Ed. Engl. 8, 781 (1961).

[7] F.A. Cotton, Chemical Applications of Group Theory, Wiley-Interscience, New York 1971.

[8] L.A. LaJohn, P.A. Christiansen, R.B. Ross, T. Atashroo, W.C. Ermler, J. Chem. Phys. 87, 2812 (1987).

[9] L.F. Pacios, P.A. Christiansen, J. Chem. Phys. 82, 2664 (1985).

[10] T.H. Dunning Jr., P.J. Hay, in: Methods of Electronic Structure Theory, Ed. H.F. Schaefer III, Plenum Press, New York 1977, p. 1.

[11] F.B. Van Duijneveldt, IBM Tech. Res. Rep. RJ 945 (1971).

[12] M.W. Schmidt, K.K. Baldridge, J.A. Boatz, S.T. Elbert, M.S. Gordon, J.H. Jensen, S. Koseki, N. Matsunaga, K.A. Nguyen, S. Su, T.L. Windus, M. Dupuis, J.A. Montgomery, J. Comput. Chèm. 14, 1347 (1993).

[13] Tables of Interatomic Distances and Configuration in Molecules and Ions, Ed. L.E. Sutton, The Chemical Society, London 1958.

[14] F.A. Cotton, W.H. Ilsley, W. Kaim, Inorg. Chem. 18, 2177 (1979). 